\title{
Lawton, Michael T: Seven aneurysms: Tenets and techniques for clipping
}

\author{
Thieme 2011, ISBN 978-1-60406-054-6
}

\section{Philippe Bijlenga}

Published online: 19 June 2012

(C) Springer-Verlag 2012

The first time I saw this book, I thought, "Again a book on intracranial aneurysm microsurgery," but I rapidly changed my mind while reading this masterpiece that synthesizes and modernizes concepts taught by G. Yaşargil.

The essence of the book is to systematically review the most pertinent aspects of aneurysm surgery. The book itself illustrates all qualities required for a neurovascular surgeon. It is well organized, concise, and extremely well illustrated. Great care has been given to detail. Extremely important and often forgotten aspects regarding the management of the surgeon's and the team's emotions are discussed with humility and humanity in a well-balanced equilibrium.

Seven aneurysm locations were selected that the author claims should be saved for microsurgical clipping. I would argue that the list is slowly fading out, but agree that surgeons will still be facing vascular lesions for a long time.

A first section reviews the most important general principles, defines landmarks, and reveals simple rules to be known, remembered, and mastered to excel in the art of neurovascular surgery.

A set of four surgical approaches allowing access to all seven types of aneurysms is clearly presented.

The third section reveals the particular microsurgical anatomy, and recommended strategies for dissecting and techniques for clipping aneurysms in the seven selected typical locations. Each chapter is extensively illustrated by sketches and high-quality operating field photographs.

Michael T. Lawton has succeeded in transforming his experience into teachable lessons and insights, guiding neurosurgeons to excel in their own practice for the benefit of aneurysm patients.

Conflicts of interest None.
P. Bijlenga $(\bowtie)$

Hôpitaux Universitaire de Genève,

Genève, Switzerland

e-mail: philippe.bijlenga@hcuge.ch 